\title{
Relationship Between Voluntary Medical Male Circumcision and HIV Reduction: A Critical Review
}

\author{
Owino B. Kevin \\ School of Humanities and Social Sciences, Kenyatta University, Nairobi, Kenya
}

Email address:

kowinobaraza@gmail.com

\section{To cite this article:}

Owino B. Kevin. Relationship Between Voluntary Medical Male Circumcision and HIV Reduction: A Critical Review. International Journal of HIV/AIDS Prevention, Education and Behavioural Science. Vol. 3, No. 1, 2017, pp. 1-6. doi: 10.11648/j.ijhpebs.20170301.11

Received: August 21, 2016; Accepted: February 13, 2017; Published: March 2, 2017

\begin{abstract}
In Kenya, male circumcision among earlier non practicing communities, like the communities from Western Kenya gained popularity in 2008. This is when the voluntary medical male circumcision was introduced as one of the campaigns to reduce the spread of Human Immunodeficiency Virus (HIV). According to [1], as at the end of the year 2013, 792,000 males had been circumcised in the region. The purpose of this study was to investigate the relationship between voluntary medical male circumcision and HIV reduction. The study was based on already done studies and purely depended on findings of past studies. The study revealed that voluntary medical male circumcision can significantly reduce contraction of HIV. It was evident that voluntary medical male circumcision only provides partial protection from HIV infection. While some studies showed that male circumcision is statistically proven to reduce HIV contraction among men than in women. It was also noted that is not properly done, HIV can be transmitted during male circumcision. This review study recommends further study in HIV prone areas like Western Kenya to assess the specific impact of voluntary medical male circumcision commissioned by Kenya government in 2008.
\end{abstract}

Keywords: Voluntary Medical Male Circumcision, HIV Reduction, Luo Community

\section{Introduction}

HIV/AIDS has been and continue to be a problem to many Nations; especially in Africa. HIV/AIDS is considered the world's leading infectious killer recording an estimate of 36 million deaths since 1981 when the first case was reported [11]. According to World Health Organization (WHO), 2.1-2.6 million died of HIV/AIDS related illnesses worldwide in 2006. In 2012, this number declined to 1.6-1.9 million [2]. Research shows that the number of people living with HIV/AIDS as at 2012 was 35.3 million. Most of these people live in low and middle income nations. Africa records the highest number of infection with a statistic of 1 in every 20 adults living with the virus $[4,2]$

In 2004, Kenya was ranked 9th in Africa in AIDS prevalence [4]. According to [6], as at 2013, more than 1.6 million people (about six percent of the population) were living with HIV/AIDS in Kenya. About 58,000 people had died of HIV/AIDS as at the same year. These deaths led to the number of children living without parents rising to 1.1 million. It is important to note that children have not been left out. There are approximately 190,000 children living with the virus in Kenya. HIV/AIDS prevalence is highly felt in Western parts of the country recording between 20-30 per cent [9].

No cure for HIV/AIDS has been invented. To deal with the epidemic of HIV/AIDS, a number of initiatives have been put into place. The initiatives have concentrated in the following areas: preventing new infections, care, treatment and support of the already infected and care for the affected. The most popular methods for prevention of new infection is abstinence, maintain one sex partner or use of condoms. For care, treatment and support, the commonly used methods are use of antiretroviral therapy, maintenance of healthy eating habit and care for the infected. Another prevention method that is gaining popularity is male circumcision. Research shows that male circumcision can reduce HIV infection by $60 \%$ [12].

Male circumcision is the removal of foreskin of the penis [15]. Male circumcision has been a common practice in many African communities. In Kenya, the practice is used as an initiation rite of passage among the Luhya, Kisii, Kalenjin, Maasai among other groups. However, this practice is not popular among the Luo community. Male circumcision has 
been embraced as one of the interventions by the National HIV Prevention Program. In the year 2008, the Ministry of Public Health and Sanitation introduced voluntary male circumcision to reduce HIV/AIDS infection. This method majorly targeted the uncircumcised part of the population, majorly the Luo community. Since 2008, the Luo community has gradually adopted the practice.

HIV/AIDS has remained one of the greatest public health challenges, not just in Kenya but globally. Numerous researches have been done on the possible cure of HIV. However, it has not been possible to invent a cure for the disease. Since there is currently no cure for the infection, the only option to contain the vise is to prevent new infections and care for infected individuals. The Kenyan government has not been left out in the fight against HIV/AIDS. One of the initiatives adopted by the Kenyan government is the introduction of voluntary male circumcision campaign in 2008. A number of researches have been done on HIV/AIDS. Much of this research has focused on the trends of HIV/AIDS and effects and impacts of HIV/AIDS on societies. A few studies have been done to relate the male circumcision and HIV/AIDS reduction.

This study was limited by the fact that HIV AIDS is perceived to be a very sensitive disease, associated with adultery in the Luo Community. Secondly, the study was limited by time constraints. However, since a number of studies have been conducted on male circumcision and HIV/AIDS, there was readily available secondary data to analyze and make conclusions in the study. The study therefore was based on critical analysis of existing findings.

\section{Literature Review}

\subsection{Theoretical Framework}

There are two theories that have been used to explain behavior. These theories have been adopted by researchers to explain the relationship between individual behaviors that can put them to risk of contracting HIV. Two theories; the Theory of Reasoned Action and the Theory of Planned Action will be used in this study.

\subsubsection{The Theory of Planned Behavior (TPB)}

The theory of planned action was put forward by [11]. Ajzen's theory of planned behavior points out that planned behaviors are triggered by intentions influenced by intentions that are founded on individual's attitude towards a behavior, subjective norms and individual's perception towards a given behavior [12]. Attitude is defined by [14] as individual's positive or negative feelings about performing an act or behaving in a particular way. Ajzen's theory of planned behaviour is majorly applied in health sciences to understand human behaviours [9]. Individual behavior is predicted intentions that are an outcome of individual's attitude and belief system as shown in figure 1

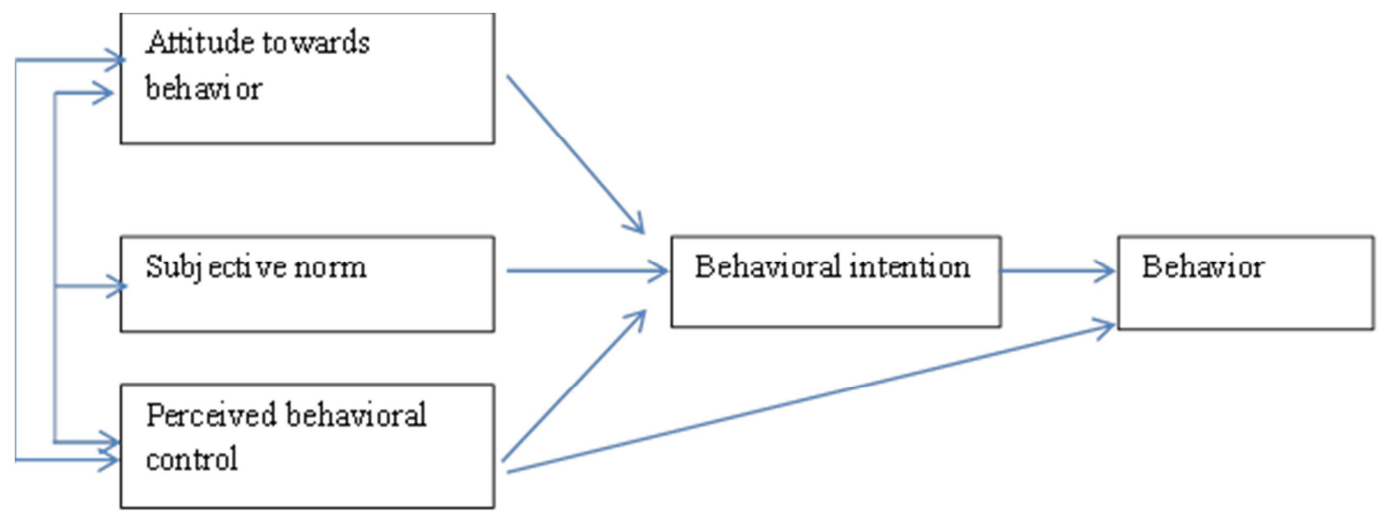

Figure 1. Ajzen's Theory of Planned Behavior.

The theory of planned behavior can be used to predict behaviours such as academic misconduct, gambling behaviours, adolescents' use of social networking sites and other behaviours such as drug abuse and irresponsible sexual behaviour. This theory can be used to understand what motivates individuals into irresponsible sexual acts especially the informed individuals.

\subsubsection{Integrated Behaviour Model}

This is an integration of the theory of reasoned action and the theory of planned action. This model predicts that behaviour that brings a health risk is proximately related to personal intentions and is shaped by individual attitudes. Such behaviours are controlled by behavior specific knowledge and attitudes, subjective social behaviours and perception of self-control Cited by [8]. Cited by [1], [17] points out that peer influence has an influence on behavior intention and consequently the actual HIV risk. In addition to peer influence, family instability, educational deficits and mental illness, substance and alcohol use and self-efficacy for HIV prevention are also blamed for such behaviour.

\subsection{Male Circumcision}

Male circumcision is the removal of the fore skin of the penis [19]. According to [13], male circumcision is the surgical removal of all or part of the fore skin of the penis as a part of a religious ritual conducted normally after birth, as cultural practice of initiation to manhood or as a medical practice related to infection or injury. 
According to [15], male circumcision should not be imposed on anyone. It should be a voluntary process. It is acceptable to have programs and campaigns encouraging adults into male circumcision, however, the programs should not lure children who are unable to make informed decisions into the act. They argue further that the children are sexually inactive neither do they engage in sexual behavior. To them, cutting off of the fore skin violates an individu7al's right to bodily integrity and self-determination. Other than the ethical concerns, [19] argues that male circumcision is a very costly affair. In most African societies, the practice is associated with celebrations that costa between Kenya shillings 10, 000 to USD 200. However, Despite the initiatives taken by the government of Kenya to ensure free circumcision services, traditional male circumcision still remains popular especially in the rural set ups.

Male circumcision is considered a general cultural practice in Africa; it is common in eastern and southern regions of the continent. Bantu groups such as Bukusu, Gisu, Bagisu and Bagesu are among the circumcising African communities. However, not all Bantu groups circumcise. Soga and Gandagoups of the Bantus do not practice the act. Similarly, some Nilotic groups such as Sabei and Nandi circumcise while others like Luo and Teso do not [13]. Most of these communities practice the act as cultural practice of initiation and passage. [1] categorized male circumcision into: traditional male circumcision and clinical male circumcision. Traditional male circumcision is based on the African cultural practices that looked at male circumcision as an initiation process. On the other hand, clinical male circumcision is based on medical practices where the removal of the fore skin is done by qualified health practitioners. According to [17], most African men are circumcised through traditional male circumcision. Clinical circumcision is mainly done by the middle class members of the society living in urban areas. In 2008, the government of Kenya introduced voluntary medical male circumcision across the country in all medical facilities. The service is currently free and accessible in all parts of the country.

\subsection{HIV/AIDS}

The first case of HIV aids was reported in 1984 in Kenya [14]. Since then the epidemic has spread to be a challenge in the entire country. The human body depends on the immune system to fight infections. HIV interferes with the immune system of the body by using the white blood cells to replicate itself. When an individual is infected by HIV, his or her immune system is affected rendering the individual vulnerable to other common diseases like tuberculosis and malaria [10]. There are different types of HIV. The first type is HIV-1 which is common in Kenya as well as other parts of the world. The second type is HIV-2 which has a lower transmission rate as compared to HIV-1. HIV can also be categories into sub groups as type A, B, C and D. type A is common in Africa, type B in America and Europe while types $\mathrm{C}$ and $\mathrm{D}$ are common in Africa and India [11].

According to [4], caring for HIV/AIDS victims is a very involving tasks that not only physical attention but also emotional attachment and concern. These cases are more demanding when the victims are aging. Other than the individual challenges that are specific to the patients, there are external challenges that negatively impact on the caretakers efforts. Such challenges include: limited coverage of education and awareness programs, inadequate surveillance system, health system constraints, financial constraints and socio-cultural challenges [10]. According to [11], most HIV patients cannot tolerate the side effects of the disease or follow the drug regimes. Home based care are therefore recommended to ensure HIV infected patients are closely monitored.

HIV infection can be through: sharing of drug needles or syringes, sexual contact with HIV positive individuals or during birth when an HIV positive gives birth without proper care. Other sexually transmitted diseases like syphilis and gonorrhea can also enhance the chances of one contracting HIV [11]. In Africa, HIV contraction is majorly through penile-vaginal sex [16]. A report produced by Ministry of Health, Kenya identified three main transmission methods: heterosexual contact, perinatal transmission; transmission of the virus from the mother to the new born and blood transmission especially during blood transfusion [16].

The following are the common symptoms of HIV/AIDS: swollen glands that take more than three months, frequent fever and sweats, skin rashes, short term memory loss, cough and shorter breaths, slow growth and frequent illness in children, difficulty in swallowing, vision loss, weight loss, fatigue, severe headache and reduced immunity.

Despite the numerous attempts by research and medical institutions on the cure for HIV/AIDS, no permanent cure has been invented. However, there are recommended methods that can be used for 'treatment'. The first method is Antiviral Therapy which involves the use of antiretroviral drugs to inhibit the spread of HIV cells [12]. Despite the effectiveness of this method, antiviral therapy is associated with side effect ranging from: fever, nausea, diarrhea, vomiting, fatigue, headaches and skin rush which make it unpopular among some HIV patients [17]. It is important to note that this method is very expensive and may be beyond the reach of the common citizens if not subsidized. Other treatment methods include: pain treatment, treatment for other infections like cancer and TB and treatment for symptoms [8] and [9]

A part from treatment methods, there are methods that can be used to reduce or prevent new infections. New HIV contraction can be prevented through abstinence from sexual intercourse, maintenance of one sex partner and practice of due care and hygiene. In addition to the prevention methods, the chances of contracting HIV can be reduce through use of condoms, upholding high level of moral standards and through male circumcision. For already infected individuals, further spread of the virus may be reduced by the individuals maintaining one sexual partner. According to [6], HIV prevention approaches include: care, support and treatment services and HIV testing and counseling.

A report produced by the [7] identified a number of 
interventions that can be used to prevent transmission of HIV. The interventions are: interventions to prevent heterosexual transmission, promotion of abstinence before marriage and faithfulness to one partner after marriage, promoting voluntary counseling and testing, promoting availability and use of condoms, controlling sexually transmitted diseases, preventing infection among young people and interventions to prevent mother to child transmission during birth.

\section{Methodology}

This review purely focused on past literature to assess the influence of voluntary medical male circumcision on HIV reduction. Literature was reviewed from various studies conducted by researchers a cross the world. A critical review of the empirical literature is presented in section 4 below.

\section{Male Circumcision and HIV/AIDS Reduction}

According to [7], circumcision cannot prevent the spread of HIV. Just like the uncircumcised men, circumcised men can contact, transmit and die from HIV. To them, transmission of HIV is caused by irresponsible sexual behavior such as having multiple sex partners and failure to use condoms and use of contaminated instruments such as needles. According to [18], male circumcision has significant effect in reduction of HIV prevalence. In Africa, male circumcision is practiced mainly either as a cultural or religious practice. Studies conducted among communities practicing and not practicing male circumcision showed the circumcised groups had lower HIV prevalence. Male circumcision was reported to have a protective effect on HIV contraction. These studies showed the uncircumcised as a vulnerable group as far as contraction of HIV is concerned [11].

Most observational reviews have proved there is a positive relationship between male circumcision and HIV infection. A part from the direct impact of male circumcision, the circumcision ceremonies can be used as sensitization platforms to educate the public on good morals and hygienic sexual behaviours that in turn will reduce irresponsible sexual behaviours that enhance HIV transmission. Numerous observational studies show that circumcised men have lower levels of HIV infection as compared to their uncircumcised counterparts [13]. Generally, HIV prevalence is lower among communities that practice male circumcision. For instance, the prevalence of HIV in southern Asian countries where men are circumcised record lower HIV infections as compared to other parts of the continent [5]. Similarly, male circumcision is efficacious in reducing sexual transmission of HIV. It offers partial protection against transmission of HIV by up to $60 \%[1]$

As discussed by [17], three studies conducted in Africa showed that male circumcision reduces contraction of HIV. [6] further pointed out that different studies have shown different results. Some reported higher rate of HIV contraction among the uncircumcised while others showed the opposite. Figure 2 show a summary of some of the studies conducted in the respective countries in 2005 .

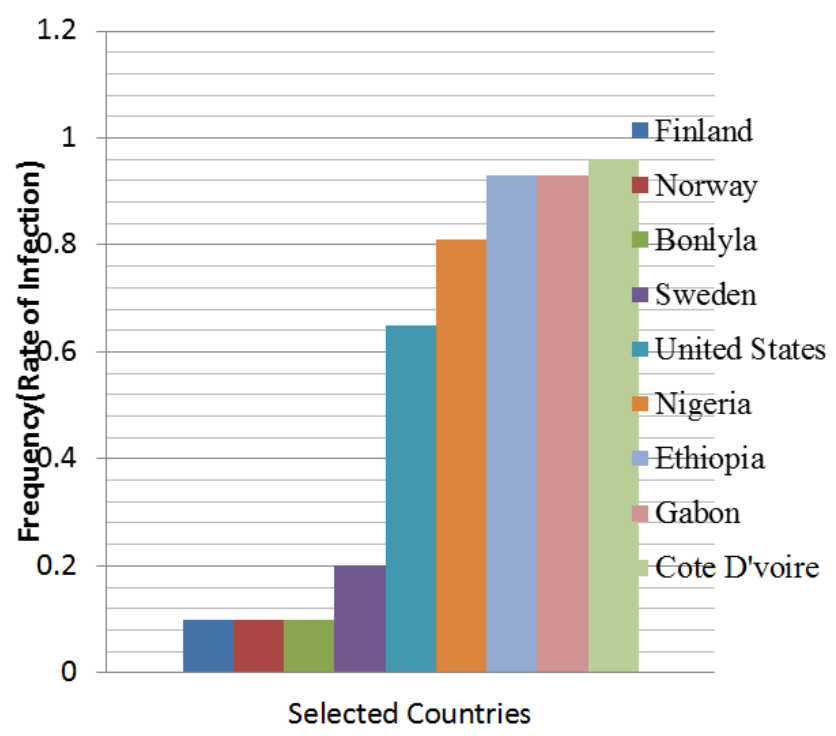

Figure 2. Circumcision \& HIV Infection Rates in Selected Countries.

A study that focused on male circumcision and heterosexual transmission of HIV in Africa was conducted in 2007. The study included 19 cross sectional studies, 5 case-control studies and 3 cohort studies. These studies showed that male circumcision has a substantial effect on reduction effect on HIV contraction. They showed a reduced level of $44 \%$ in circumcised men [3]. In other studies conducted in Uganda, the results showed reduced risk on contraction of HIV aids among HIV negative circumcised men engaging in sexual act with HIV infected women [5]. A study conducted by [17] showed that male circumcision has significant effect in reduction of HIV prevalence. This study showed further that male circumcision can be used to enhance growth. This is because HIV prevalence is directly correlated with economic development of a nation. This study however points out that it is important to incorporate other factors such as awareness campaigns, education and nutritional outreach if we have to deal with the issue of HIV.

A research was conducted to investigate whether male circumcision is acceptable among non-circumcising communities as an HIV reduction intervention, how gender and ethical practices are affecting the perception of the practice in non-circumcising communities, how to collaborate the clinical and the traditional male circumcision, how to consent on the best age for male circumcision, how male circumcision can be promoted to enhance acceptability without reducing adoption of other preventive like the use of condom and the specific criteria that can be used to prioritize male circumcision as HIV prevention method. The results showed that male circumcision is statistically proven to reduce HIV contraction among men [16].

A study conducted by [10] on male circumcision for HIV prevention: a prospective study of complications in clinical 
and traditional settings in Bungoma, Kenya showed that two thirds of Kenyan men are circumcised. The study concluded that male circumcision can reduce contraction of HIV by up to $60 \%$. The study further recommended that male circumcision be adopted as a strategy of reducing contraction of HIV. However, the study noted that there is need for extensive training and resources availability to ensure safe circumcision. There is also need for intensive awareness campaign and sensitization programs.

As discussed by [9], a number of studies have been done to explore the relationship between male circumcision and HIV infection. Most of these studies sought to assess the possibility of use of male circumcision as a method of reducing the transmission of HIV infection in men. Most of the studies revealed there is positive relationship between male circumcision and HIV contraction. However, some studies showed negative relationship. [10] further pointed out that a part from the practice itself reducing chances of HIV transmission, the male circumcision ceremonies can be used as platforms to where men can be taught hygienic sexual practices such as monogamy and penile hygiene.

A study conducted by World Health Organization and [1] titled New Data on Male Circumcision and HIV Prevention: Policy and Programme Implications concluded that male circumcision reduces sexual transmission of HIV both from infected male to female and infected male to female. The research further showed male circumcision as a practice in itself can cause HIV transmission. Professionally conducted male circumcision reduces HIV transmission by up to $60 \%$. The study recommended that male circumcision should be recognized as an efficacious intervention for HIV prevention and that the practice should be recognized as an additional strategy for prevention of heterosexually acquired HIV infection in males. However, the study outlined that male circumcision only provide partial protection from HIV infection. It is therefore important that there is effective communication to sensitize the communities on the importance and limitations of male circumcision as a strategy of reducing HIV infection. This study recommended that further studies should be conducted to clarify the risks and benefits of male circumcision as a strategy or reducing HIV transmission from HIV male to females and to recommend how safer male circumcision can reach rural areas.

\section{Conclusion}

From critical review of empirical literature, it was evident that voluntary medical male circumcision can significantly reduce contraction of HIV. It was evident that voluntary medical male circumcision only provides partial protection from HIV infection. Some studies showed that male circumcision is statistically proven to reduce HIV contraction among men than in women. Where male circumcision is done in non-hygienic conditions, cases of HIV transmission have been reported. It is therefore recommended that male circumcision is done in a medical facility by qualified medical practitioners. This study recommend further study in
HIV prone areas like Western Kenya to assess the specific impact of voluntary medical male circumcision commissioned by Kenya government in 2008 .

\section{References}

[1] Bureau, P. R. (2009). HIV/AIDS and Older Adults in the United States. Today's Research on Aging. Programs and Policy Implications. Issue 18, December. Population Reference Bureau.

[2] Ginsburg, H. (2010). Ajzen's Theory of Planned Behavior Applied to the Use of Social Networking by College Students.. Honors Thesis. Presented to the Honors Committee of Texas State University-San Marcos.

[3] Greene, K. H. (1997). A Test of the Theory of Action in the Context of Condom Use and Aids. Communication Reports, Volume 10 No. 1.

[4] Hale, J. L. (2000). The Theory of Reasoned Action.

[5] Hayes, R. H. (1999). Meta-analysis of the Relationships between Male Circumcision and HIV Infection. Presented at the 13th meeting of the ISSTDR. July 11-14, 1999. Denver, CO, USA.

[6] Ministry of Health, Kenya. (2011). AIDS in Kenya. Nairobi: Sixth Edition.

[7] Aids Info (2014). Guidelines for Prevention and Treatment of Opportunistic Infections in HIV Infected Adults and Adolescents. Retrieved September 9th, 2014, from AIDS Info: http://aidsinfo.nih.gov/contentfiles/lvguidelines/Adult_OI.pdf.

[8] Mark, J. (1997). Aspects of Male Circumcision in Sub-equatorial African culture history. Health Transition Review, Supplement to Volume 7, 337-359.

[9] Nyaga R. K. (2014). HIV/AIDS in Kenya: A Review of Research and Policy Issues.. KIPPRA Discussion Paper No. 38 June 2004.

[10] World Health Organization. (2014). Number of deaths due to HIV/AIDS. Retrieved September 7th, 2014, from http://www.who.int/gho/hiv/epidemic_status/deaths_text/en/.

[11] Polgar, M. (2009). HIV Prevention for Youths in Foster Care: Understanding Future Orientation and Intended Risk Behaviors. Journal of HIV/AIDS \& Social Services, 8: 397-413.

[12] Havard Medical School. (2009). Male circumcision and AIDS: The Macroeconomic Impact of a Health Crisis. Havard Business.

[13] Siegfried N. (2003). Male Circumcision for Prevention of Heterosexual Acquisition of HIV in men - a Cochrane Review. MRC Policy Brief. No. 2, July 2003.

[14] Siegfried N. (2003). Male Circumcision for Prevention of Heterosexual Acquisition of HIV in Men - a Cochrane Review. MRC Policy Brief No. 2, July 2003.

[15] CDC. (2008). Male Circumcision and Risk for HIV Transmission and Other Health Conditions: Implications for the United. CDC HIV/AIDS Science Facts.

[16] UNAIDS. (2013). AIDS by the Numbers. Retrieved September 7th, 2014, from UNAIDS:

http://www.unaids.org/en/media/unaids/contentassets/docume nts/ naidspublication/201JC2571_AIDS_by_the_numbers_en.pdf. 
[17] USAID. (2013). USAID Kenya HIV/AIDS.. Retrieved September 7th, 2014, from USAID:

http://www.usaid.gov/news-information/fact-sheets/usaid-ken yahivaids.

[18] Van Howe, S. (2005). HIV Infection and Circumcision: Cutting Through the Hyperbole. The Journal of the Royal Society for the Promotion of Health, 125 (6): 259-65.
[19] Wasti, S. P. (2009). Issues and Challenges of HIV/AIDS Prevention and Treatment Programme in Nepal. Global Journal of Health Science, 1 (2), 62-72.

[20] Williams, I. C. (2014). British HIV Association guidelines for the treatment of HIV-1-positive adults with antiretroviral therapy. 